\title{
The ENVELOPES of BEAM MOTION in the CHARGED PARTICLE CYCLIC ACCELERATOR
}

\author{
Virchenko Yu.P. \\ Single Crystal Institute, UA-310001, Kharkov, Ukraine \\ Grigor'ev Yu.N. \\ Kharkov Institute of Physics \& Technology, National Science Center, \\ UA-310108, Kharkov, Ukraine
}

\begin{abstract}
.
The method for calculation of the beam motion envelopes in the six-dimensional phase space of charged particle moving in a cyclic accelerator is proposed. It is based on the invariance of the special quadratic form depending on the dynamic variables relatively to the shift along the particle trajectory. The envelopes are expressed as the explicit functions of the form coefficients.
\end{abstract}

\section{Introduction}

The classical problem for development of cyclic accelerator of charged particles is the calculation of the envelopes of the particle beam with respect to different dynamic variables $u_{l}(\theta), l=1, \ldots, 6$, which are the components of the state vector $u(\theta)$ of a charged particle in the point with fixed accelerator azimuth $\theta$. This problem is actual as before (see, for example [1-3]). The envelopes determine the maximum possible values of the respective dynamic variables in any point $\theta$ of the accelerator ring and therefore they determine various important physical characteristics of the beam: transverse sizes, divergence and energy spread [4,5]. In the present communication we propose the method for calculation of the envelopes in terms of the evolution matrix elements.

\section{Evolution matrix}

In general case the components of the state vector may be expressed by the set of the following values $(x, \dot{x}, \delta, \eta, z, \dot{z})$ [4], i.e. $u_{1}(\theta)=x(\theta), u_{2}(\theta)=\dot{x}$, and so on. Here $x, z$ are the particle deviations along the normal and the binormal to the closed orbit respectively; $\delta=\left(E-E_{s}\right) / E_{S}, E$ is particle energy, $E_{s}$ is the energy equilibrium value, $\eta=\left(\varphi-\varphi_{s}\right)$ is phase deviation from the equilibrium value; $\dot{x}, \dot{z}$ are the rates connected with the coordinates $x, z$ respectively. The charged particle dynamics in the cyclic accelerator in the one-particle approximation with negligibly small random perturbations is described by the set of linearized equations $[4,5]$

$$
\dot{\mathrm{u}}(\theta)=A(\theta) \mathrm{u}(\theta),
$$

where the $6 \times 6$ - matrix $A(\theta)=\left(A_{l, m}(\theta)\right), l, m=1, \ldots, 6$ is the periodic function with respect to $\theta$ and it has a definite structure with the dependence on $\theta$ defined by the given accelerator design. However, the concrete form of matrix $A(\theta)$ has no importance for the below proposed method.

Determine the evolution matrix $M(\theta)$ for the above linear system by the relationship $M(\theta) \mathrm{u}(0)=\mathrm{u}(\theta)$. This matrix is the unique solution of the equation

$$
\dot{M}(\theta)=A(\theta) M(\theta)
$$

with initial condition $M(0)=1$. Since the dependence $A(\theta)$ is piecewise constant and it is periodic respective to $\theta[4,5]$, then the matrix $M(\theta)$ can be always determined explicity on the basis of quite tedious calculations for the specified accelerator design. Further, the matrix $M(\theta)$ will be assumed known. To determine the motion envelope with respect to the $u_{l}$ - phase coordinate it is necessary to calculate $\max \left[M_{l k}(\theta) u_{k}(0)\right]$ where the maximum is determined by the exhaustion of all possible initial data $u_{k}(0), k=1, \ldots, 6$, which the beam particles possess at certain fixed initial moment. This calculation procedure is very inconvenient and, therefore, it is preferable to obtain the formulae for envelopes directly in terms of matrix $M(\theta)$. Such formulae are known for the case of the independent $x$-oscillations provided that the energy homogeneity takes place [6].

\section{Invariant quadratic form}

Introduce the matrix $G(\theta)=M(\theta) M(\theta)^{+}(+$denotes the matrix transposition), which according to Eq.(2) satisfies to the equation

$$
\dot{G}(\theta)=A(\theta) G(\theta)+G(\theta) A(\theta)^{+}
$$

and to $G(0)=1$. The matrix $M(\theta)$ is reversible because $d(\ln \operatorname{det} M(\theta)) / d \theta=\operatorname{Sp} d(\ln M(\theta)) / d \theta=\operatorname{Sp} M^{-1}(\theta) M(\theta)=$ $\operatorname{Sp} A(\theta)$ and hence

$$
\operatorname{det} M(\theta)=\exp \left\{\int_{0}^{\theta} \operatorname{Sp} A(\theta) d \tau\right\} \neq 0
$$

Then the matrix $G(\theta)$ is reversible and it may be possible to introduce the quadratic form $I(\theta)$ depending on the state vector $u(\theta)$

$$
I(\theta)=\left(\mathrm{u}(\theta), G^{-1}(\theta) \mathrm{u}(\theta)\right) .
$$

Using the differentiation formula $d G^{-1}(\theta) / d \theta=$ $-G^{-1}(\theta) \dot{G}(\theta) G^{-1}(\theta)$ and Eqs.(1),(3), we find that value $I(\theta)=$ $I_{0}$ is a formal invariant relative to shift along the trajectory. So, the particle moves on the six-dimensional surface the shape and the location of which in the six-dimensional phase space are regulated by matrix $G^{-1}(\theta)$ and therefore it is changed with $\theta$. The surface is formed by the trajectories with the initial vectors $u(0)$ which give the same value of the invariant, $I_{0}=(\mathrm{u}(0), \mathrm{u}(0))$. Since the surface under consideration is the sphere at time $\theta=0$, then it is closed and simply connected at any time moment. 
Moreover, since the surface with lesser value of the invariant $I_{0}$ at time $\theta=0$ is putted into the surface with greater value, then this relation between these surfaces is conserved during the motion. Due to this we may set up a problem of the trajectory envelopes corresponding to the initial data $u_{0}$ which are inside the fixed six-dimensional sphere $(u(0), u(0))=I_{0}$. At a fixed moment $\theta$ all these trajectories are contained inside the sphere in the $u_{0}$-space which is formally described by the equation

$$
\left(\mathrm{u}\left(\theta, \mathrm{u}_{0}\right), G^{-1}(\theta) \mathrm{u}\left(\theta, \mathrm{u}_{0}\right)\right)=I_{0}
$$

with the vector $u_{0}$ as the surface parameter. Passing on the parametric description on the base of vector $u=u\left(\theta, u_{0}\right)$ we find that this surface is the face of the six-dimensional ellipsoid

$$
\left(\mathrm{u}, G^{-1}(\theta) \mathrm{u}\right)=I_{0}
$$

at each moment $\theta$. It is true because the above coordinate transformation is affine due to the linearity of the Eq.(2). Thus, the desired envelopes are formated by means of the motion of the surface (5).

Notice, that for the system with one degree of freedom which describes the $x$-oscillations one would show that $G_{22}(\theta)=$ $\beta(\theta), G_{12}(\theta)=-\dot{\beta}(\theta) / 2, G_{11}(\theta)=\gamma(\theta)$ where $\gamma$ is determined from the condition $\operatorname{det} G(\theta)=1$. In this case the using of Eq.(4) gives the expression

$$
I=\gamma \dot{x}^{2}-\dot{\beta} x \dot{x}+\beta \dot{x}^{2}
$$

coinciding with Courant-Snyder invariant [6]. Consequently, the surface in this case is the ordinary ellipse.

\section{Envelopes of beam motion}

We consider now the particle motion neglecting the friction connected with the particle radiation. Then Eq.(2) represents the canonical Hamiltonian system for which $\operatorname{Sp} A(\theta)=0$, i.e. $\operatorname{det} M(\theta)=1$ [7], and the phase volume containing inside the surface (5) is constant due to Loiuville's theorem. We assume further that system (2) is stable, i.e. the case when eigenvalues of the monodromy matrix $M(T)$ ( $T$ is the period $A(\theta+T)=A(\theta))$ have unit modulus and the set of theirs is divided on mutually conjugate pairs. The typical trajectory of the particle motion is almost periodic in general case, i.e. the eigenvalues are immenconsurable. However each trajectory moves on accompanying ellipsoid (5). In turn the matrix $G(\theta)$ is not periodic also in general case, $G(T) \neq G(0)=1$, i.e. the ellipsoid (5) does not coincide with itself after a lapse of the period. It is connected with that the monodromy matrix is not unitary in general case due to its eigenvectors are not mutually orthogonal. Then the desired envelopes are the envelopes of all possible shifts $\theta \rightarrow \theta+n T, n=1,2,3, \ldots$ of the surface (5). Since we want to determine separately the envelope relative to each dynamic variable $u_{l}$, then it is suitable to divide the solution of this problem on two stages. On first stage we determine the maximum value $\bar{u}_{l}(\theta)=\max \left[u_{l}(\theta)\right]$ of the variable $u_{l}(\theta)$ for all trajectories with the initial values $u_{0}$ inside the sphere (5) at $\theta=0$. On second stage we must calculate the maximum value $\tilde{u}_{l}$ of the function $\bar{u}_{l}(\theta)$ on all possible shifts of the $\theta$,

$$
\tilde{u}_{l}=\sup \left\{\bar{u}_{l}(\theta+n T), n=0,1,2,3, \ldots\right\}
$$

This value is the desired envelope for the dynamic variable $u_{l}(\theta)$.

First stage of the above described procedure is reduced to the simple geometrical problem connected with the ellipsoid. We give it solution below. Under above considerations to calculate the values $\bar{u}_{l}(\theta)$ it is sufficient to find the value $\bar{u}_{l}=\max \left[u_{l}\right]$ where the maximum is computed for all points of the enveloping ellipsoid (5). Calculate, for example, the value $\bar{u}_{1}$, determining the maximum of $u_{1}$ by varying $u_{2}, \ldots, u_{6}$ over the ellipsoid surface. In this case we may consider $u_{1}$ to be the implicit function of $u_{2}, \ldots, u_{6}$ parameters. Since in Eq.(5) the $\theta$ is fixed and, hence, we do not specify below the dependence on $\theta$. Denote $P \equiv G^{-1}(\theta)$ and by symbol $P^{\prime}$ the truncated $5 \times 5$ - matrix which is obtained from $P$ by crossing out the first line and the first column. Denote also $Q \equiv\left(P^{\prime}\right)^{-1}$. In all below formulae the summation respective to repeating indexes is performed from 2 to 5. By differentiating Eq.(5) on $u_{s}, s=2, \ldots, 5$ respectively and requiring the maximality of $u_{1}$, i.e. $u_{1} \rightarrow \bar{u}_{1}, \partial u_{1} / \partial u_{s}=0, s=2, \ldots, 5$, we obtain $u_{l}=Q_{l s} P_{s 1} \bar{u}_{1}, l=2, \ldots, 5$. The substitution of these $u_{l^{-}}$values in (5) gives the equation for the $\bar{u}_{1}$ - calculation

$$
I_{0}=\bar{u}_{1}^{2}\left[P_{11}-r\right], r=P_{1 k} Q_{k m} P_{m 1},
$$

where we applied the identity

$$
P_{l m} Q_{m k}=P_{l m}^{\prime} Q_{m k}=\delta_{l k} ; l, k=2, \ldots, 5 .
$$

Now we transform the expression in square brackets. It follows from the definition of the $P$ - matrix that

$$
\begin{gathered}
G_{1 k} P_{1 m}+G_{k l} P_{l m}=\delta_{k m}, G_{11} P_{11}+G_{1 k} P_{1 k}=1, \\
G_{1 k} P_{11}+G_{k l} P_{l 1}=0, k, m=2, \ldots, 5
\end{gathered}
$$

The first equation shows that $r=P_{1 m} Q_{m k} P_{k 1}+G_{1 k} P_{1 k} r$. Using second and third equations we obtain

$$
r G_{11} P_{11}=P_{1 k} G_{k m} P_{m 1}=-P_{1 k} G_{1 k} P_{11}=\left(1-P_{11} G_{11}\right) P_{11} .
$$

Substituting the expression $r=1 / G_{11}-P_{11}$ in Eq.(7) and recovering the dependence on $\theta$, we derive the final formula

$$
\bar{u}_{1}(\theta)= \pm\left(I_{0} G_{11}(\theta)\right)^{1 / 2} .
$$

The calculations performed are related literally for every dynamic variable $u_{l}$ and therefore the general formula is valid

$$
\bar{u}_{l}(\theta)= \pm\left(I_{0} G_{l l}(\theta)\right)^{1 / 2}
$$

Eq.(8) gives the general expression for $\bar{u}_{l}(\theta)$ obtaining on first stage of the above described calculation procedure. Three almost periodic functions $G_{l l}(\theta), l=1,2,3$ can be called the generalized magnetooptic functions of the accelerator structure.

Pass on the second stage of the envelope computation. If the monodromy matrix is unitary, then $\tilde{u}_{l}(\theta)=\bar{u}_{l}(\theta)$, i.e. the envelope form is determined by the Eq.(8). But the opposite case with the nonunitary monodromy matrix takes place as a rule. And we must use Eq.(6) in order to find the envelopes. Due to the almost periodicity of the matrix $G(\theta)$, the ellipsoids (5) obtained by the consequent shifts $\theta \rightarrow \theta+n T$ do not coincide with each 
other. Therefore the problem arises to find the envelope of all possible shifts mentioned for each value $\bar{u}_{l}(\theta), l=1, \ldots, 6$. To do this it may set up the following problem. Is there an ellipsoid (5) which coincides with itself after the shift $\theta \rightarrow \theta+T$, i.e. is there an initial matrix $G_{0}$ such that the matrix $G(\theta), G(0)=G_{0}$ which satisfies to Eq.(3) and to $G(\Theta+T)=G(\theta)$ ? If there are the ellipsoids with described property then we may put the initial unit sphere $\left(u_{0}, u_{0}\right)=1$ into one of them and the next question arises. Would one to elect the matrix $G_{0}$ by an optimal way when the envelope (6) generated by the motion with initial data $u_{0}$ on the sphere will coincide with the optimal ellipsoid? Answers on these questions contain the following assertions.

Theorem 1. If the system (1) is stable, then there is always the ellipsoid (5) which is invariant relative to the transformation $\theta \rightarrow \theta+T$. along the trajectories of the motion. It is determined by the matrix $G_{0}^{-1}\left(G_{0}^{+}=G_{0}\right.$, $\left.\operatorname{det} G_{0}=1\right)$ of the quadratic form coefficients. If the spectrum of the matrix $M(T)$ is not degenerate, then this ellipsoid is unique to within the isotropic dilatation.

Theorem 2. If the spectrum of the matrix $M(T)$ of the stable system is not degenerate and has not some pairs of the commensurable Flouquet exponents, then there is the unique ellipsoid enveloping all trajectories with initial data $u_{0}$ lying on the sphere $\left(\mathrm{u}_{0}, \mathrm{u}_{0}\right)=I_{0}$. This ellipsoid coincides with the invariant ellipsoid mentioned in Theorem 1. and it is circumscribed round the sphere determined by the value $I_{0}$, i.e. it is minimal among invariant ellipsoids containing the sphere.

The properties expressed by these theorems one would consider as the generalization for the linear uniform systems with periodic dependence in time of the corresponding properties of the linear autonomous Hamiltonian systems. If the oscillations of charged particle for all degrees of freedom are connected and have the immenconsurable frequencies when the system parameters are in general position, then the theorem conditions take place and we may use their assertions for the finding of the motion envelopes. In particular, on the base of Theorem 2 we may consider that the union of all ellipsoids obtained by the consequent shifts on the period $T$ from the initial sphere coincides with the circumscribed invariant ellipsoid. Therefore to find the value $\tilde{u}_{l}(\theta)$ it is sufficient to build the circumscribed invariant ellipsoid and after that to calculate the value $\bar{u}_{l}(\theta)$ for this ellipsoid on the base of Eq.(8).

Here we'll not give the complete proofs of the theorems formulated. It will be done in other publication. We'll point out only that these proofs are based on the transformation possibility of the monodromy matrix $M(T)$ to the unitary one by means of a reversible matrix $S$, which does not depend on $\theta$. This idea we illustrate below on the simple example.

Example. Consider the particular case of the linear stable Hamiltonian system for which the matrix $A$ is constant. Then $M(\theta)=\exp (A \theta)$ and the monodromy matrix $M(T)=$ $\exp (A T)$ is not unitary in general case, i.e. the condition $M(T) M^{+}(T)=1$ does not necessarily take place, since $A \neq$ $-A^{+}$and moreover the matrices $A, A^{+}$may be noncommuting. But there is the matrix $G_{0}$ such that $G_{0}^{+}=G_{0}$ and

$$
G_{0}^{-1} A G_{0}=-A^{+}
$$

It permits to build the matrix $G=M(\theta) G_{0} M^{+}(\theta)$, which does not depend on $\theta$, i.e. it coincides with the $G_{0}$ and, in particular, it is invariant relative to shift on the period. The proof of the existence of the matrix $G_{0}$ is based on the following argument. From one hand the system (1) is stable and therefore the eigenvalues of the A are imagine and in addition they form the mutually conjugate pairs. From other hand the sets of eigenvalues of the matrices $A, A^{+}$coincide with each other. Then for each $A$-eigenvalue $\alpha$ there is the $A^{+}$-eigenvalue $\alpha^{*}$ such that $\alpha+\alpha^{*}=0$. It is sufficient for the existence of the nontrivial solution of the matrix Lyapunov equation $A G_{0}+G_{0} A^{+}=0$. If the spectrum of the $\mathrm{A}$ is not degenerate, then the eigenvalue $\alpha^{*}$ is unique for each $\alpha$. and therefore the matrix $G_{0}$ is also unique due to the condition $\operatorname{det} G_{0}=1$. Notice that one would always represent the matrix $G_{0}$ in the form $G_{0}=S S^{+}$where the matrix $S$ realizes the transformation the matrix $M(T)$ to the unitary one.

\section{References}

1. D.A.Edwards, L.C.Teng, IEEE Trans. Nucl. Sci., vol.11920, No.3, p.885 (1973).

2. K.L.Brown, R.V.Servrancki, Particle Accelerators, 1991, vol.36, pp. 121-139.

3. F.Willeke, G.Ripken, Methods of Beam Optics, DESY 88-114 August 1988.

4. A.A.Kolomenskij, A.N.Lebedev"A Theory of Cyclic Accelerators", Gosizdat FML, Moscow, 1962.

5. Henri Bruck, Accelerateurs circulaires de particules, Presses Universitaires de France 1966.

6. B.Courant,H.Snyder. Theory of the Alternating- Gradient Synchrotron. Ann.of Phys. 3, 1-48 (1958).

7. V.A.Jakubovich, V.N.Starzhinskij, Linear Differential Equations with Periodic Coefficients and their applications, Nauka, Moscow, 1972. 